\title{
The Role of Multifactorial Contribution in Carpal Tunnel Syndrome Occurrence
}

\author{
Putu Feryawan Meregawa', John Nolan ${ }^{2}$ \\ ${ }^{1}$ Departement of Orthopaedic and Traumatology, Medical Faculty, Udayana University-Sanglah General \\ Hospital Denpasar, Indonesia \\ ${ }^{2}$ Faculty of Medicine, Udayana University, Denpasar, Indonesia
}

Corresponding Author: Putu Feryawan Meregawa

\begin{abstract}
As one of the common peripheral neuropathies, carpal tunnel syndrome (CTS) is accountable for the majority of typical hand pain and functional disturbance in median nerve innervation. The median nerve compression may cause some uncomfortable sensations including pain, numbness, tingling, and strength loss which also depends on the severity of the condition. Many factors could contribute to CTS occurrence. Several risk factors are thought to be in charge in CTS progressions, such as body mass index (BMI), gender, pregnancy, and biomechanical exposures combination is significantly explained as the major component in suffering CTS. Clinicians need to know the contributing risk factor to benefit the information within the implication for the treatment and reducing symptoms severity.
\end{abstract}

Keywords: carpal tunnel syndrome, risk factor, multifactor

\section{INTRODUCTION}

Carpal tunnel syndrome (CTS) is one of many common peripheral neuropathies. This condition is estimated to occur in around $90 \%$ of peripheral neuropathies. CTS is a condition that is caused by median nerve compression. This median nerve entrapment approximately affects $3 \%$ of the adult population. ${ }^{1}$ CTS is also demanding a high cost of healthcare system treatment with the addition of approximately 3,8\% believed to present with CTS. ${ }^{1,2}$ These common canalicular neuropathies are a typical reason for hand pain and functional impairment. The complaints can be varied, starting from pain, tingling, numbness, and swelling sensation on the hand and throughout median nerve distribution. The discomforts often awaken patients at night following the pain and numbness in their hands. These classic symptoms may also extend to the shoulder which could be reassured by shaking the wrist. $^{3}$ Approximately $20 \%$ of people with the main complaint of hand pain, numbness, and tingling sensations are having CTS according to physical and electrophysiological examinations. ${ }^{4}$

Feeling numbness or swelling at midnight and extremely wrist pain spreading to the shoulder are some of the classic symptoms of CTS. The dermatome with median nerve innervation produces an inconvenient perception across the thumb, index finger, middle finger, and radial side of the ring finger. ${ }^{5}$ Not only could these symptoms cause discomfort but also accumulate and form a disturbance following the strength in gripping and hand function. Muscle wasting in the base of the thumb may also occur over a long period of untreated CTS. ${ }^{6}$ People age between 40 to 60 is more susceptible to acquiring CTS. ${ }^{1,7}$ This condition also costs distinct towards the treatments, as it may consist of conservative and surgical. High cost of treatment has happened in the US as data showed the spending of around one billion US dollars each year regarding the intervention of CTS. ${ }^{8}$ 
The $4 \%$ prevalence of CTS in the general population is a warning toward disease definition and the etiology of CTS which is multifactorial and involves systemic disorder. ${ }^{9,10}$ Some systemic disorders which contribute to CTS are diabetes mellitus, obesity, and hypothyroidism. Women with the postmenopausal syndrome are also commonly affected. ${ }^{10}$

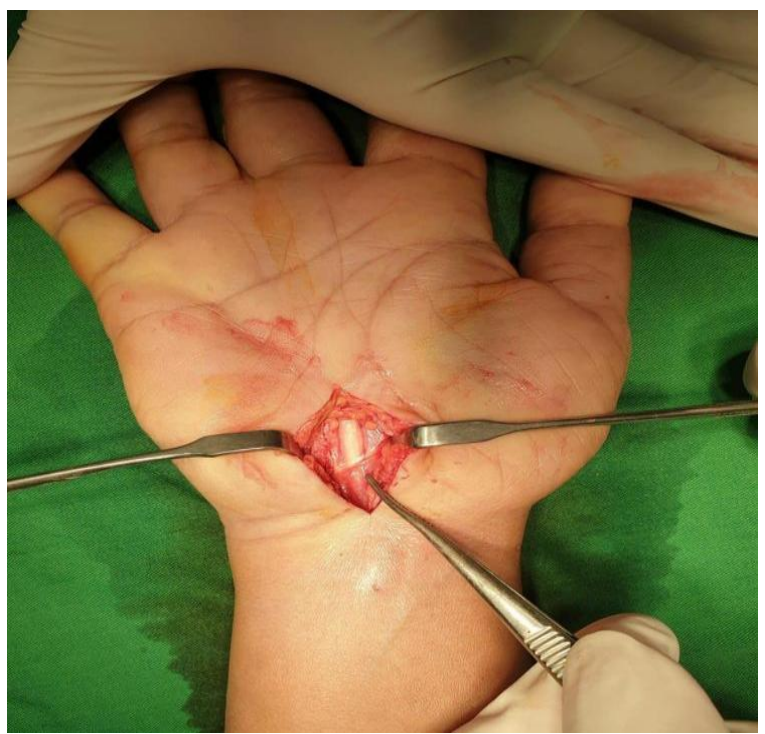

Figure 1: Open release carpal tunnel surgery to release the tension of the median nerve entrapment.

The etiology of CTS may include systemic, anatomical, idiopathic, ergonomic aspects, some indicators such as age, gender, and body mass index (BMI) associated as the risk factors of CTS. ${ }^{11}$ Higher BMI score is associated with a higher risk of developing CTS. The data also showed that obese individuals have a 2.5 times higher risk of forming CTS compared to individuals with lower BMI. On the other hand, there is no clear explanation about CTS relationship with another anthropometric measurement, such as wrist and waist circumference. ${ }^{11,12}$

As the number of obese individuals is rising, it is important to discuss the effect of obesity on CTS as it may contribute to the choice of treatment in the future. This paper is aimed to describe and review the existing literature about the current association between CTS and obesity as a major risk factor.

\section{Anatomy of Carpal Tunnel}

The Carpal tunnel is bordered with eight carpal bones and a part of a tough fibrous ligament called the transverse carpal ligament (TCL). ${ }^{13}$ Carpal tunnel provides passage for eight flexor tendons including two tendons four tendons of flexor digitorum superficialis and four tendons of flexor digitorum profunda. Two additional contents in the carpal tunnel are the flexor pollicis longus and median nerve. ${ }^{13,14}$ At the entrance of the carpal tunnel, the median nerve is located at the dorsal of palmaris longus or the middle of flexor carpi radialis (FCR) and palmaris longus. In the distal of the tunnel, the nerve is divided into six branches, including the thenar or motor branch, three palmar digital nerves, and the common palmar digital nerves that filling the second and third spaces.

The median nerve innervates three radial fingers and the radial half of the ring finger at the palmar face. The median nerve also comprises the dorsal face of the first three fingers for the last two phalanges. ${ }^{13}$ With the ten contents of the carpal tunnel, the tunnel is packed tightly. This will result in a susceptible space of compression problem if any volume inside the tunnel is increased. ${ }^{14}$

A Carpal tunnel connects the anterior part of the distal forearm with the space in the mid-palmar of the hand. Carpal tunnel forms a backward convex bony arch in the bottom as the result of carpal bone articulations. This is resulting in the dorsal side with concave in the palmar side, assembling a tunnel-like groove called sulcus carpi. ${ }^{14}$ Normal pressure in the carpal tunnel of healthy individuals is around 2 $10 \mathrm{mmHg}$ with the wrist in the neutral position. Other activities of the hand including flexion and extension provide distinctive pressure in each position. ${ }^{14,15}$

\section{Pathophysiology of CTS}

The neuropathy of median nerve entrapment is caused by the process of traction and compression. The median nerve compression causes some disturbances and 
alterations in intraneural microcirculation. This compression may also cause some myelin sheaths and axons lesions with additional connective tissue alterations. This peripheral nerve entrapment may happen due to the over-tightness of its passage through an anatomical site. Functional properties may also be altered because of the damaged nerve in the site of compression. ${ }^{16}$ Several pathophysiologic mechanisms may occur throughout the process, including the increased pressure, nerve injury, inflammation, and connective tissue alterations. ${ }^{17,18}$

As mentioned before, increased pressure in the tunnel due to changes in wrist position or external forces may lead to median nerve entrapment. ${ }^{15}$ In normal conditions, the high pressure can be withstood by the median nerve as a result of the median nerve and surrounding connective tissues' hydrostatic pressure. ${ }^{19}$ Compression due to mechanical forces may result in demyelination. This demyelination process may spread to all internodal segments causing a block in nerve transmission or neuropraxia. ${ }^{15,20}$ Small unmyelinated $\mathrm{C}$ fibers are also in charge of developing distinctive symptoms. Damaged $\mathrm{C}$ fibers induce abnormal expression of voltage-gated sodium channels (VGSC) causing an exaggeration in responses to stimuli. $^{21}$ Ischemic injury is also an important aspect of CTS. The combination between ischemic injury and continuous mechanical pressure leads to changes in the nerve fibers and causing axonal injury. These alterations can be detected through standard nerve conduction studies. In response to the repetitive motion of the wrist, it may result in inflammation or hypertrophy of the synovial of the tendons. Median nerve compression can be caused this synovial inflammation as the tendons run through the carpal tunnel. ${ }^{19}$

\section{Risk Factor of CTS}

Until now, CTS is still an idiopathic condition, however, there are several probable risk factors which may contribute informing CTS. ${ }^{22}$ Environmental risk factor contributes significantly in CTS. Posture and position may play a big role in developing CTS. $^{14}$ Occupational duties related to hand exertion duty, hand repetition, and hand vibration may result in symptoms of tingling, numbness, burning, or pain in the first three fingers. ${ }^{23}$ There are some factors included in medical factors such as external, intrinsic, and neuropathic factors. Intrinsic factors can increase the volume within the carpal tunnel, tumour and tumor-like lesions are thought to be the intrinsic factors. Extrinsic factors can be divided into two factors, the one that changes the tunnels' contour such as a direct fracture or post-traumatic arthritis. An external factor may also change the volume inside the tunnel and alter the fluid volume in the body, these include obesity, pregnancy, menopause, renal failure, hypothyroidism, the use of hormonal contraceptives, congestive heart failure. Some conditions may also worsen the median nerve without significantly escalate the pressure inside the tunnel, such as vitamin deficiency, alcoholism, diabetes, and toxin exposure. ${ }^{24}$

\section{Association between BMI and CTS}

BMI is often associated with an increased chance of acquiring CTS. It is thought to be a dose-response relationship between overweight or obesity and the occurrence of CTS. Across several studies with distinctive study designs showed a similar result toward the effect of excess body mass on CTS. ${ }^{25}$ It was found that the patients who were received the carpal tunnel release procedure are likely to be overweight or obese individuals (BMI above 25). The exact mechanism explanation of the association between obesity and CTS is still unknown. However, there are several hypotheses toward the mechanisms. The accumulation of excess fat or adipose tissue filling the carpal tunnel causing a tighter tunnel with increased intercarpal pressure. This condition may contribute to an impaired vascularisation, 
which leads to ischemia of the median nerve and other axonal injuries. Fibrosis and thickening under synovial connective tissue may also occur due to the high carpal tunnel pressure. $^{25,26}$ As one of the fundamental components of metabolic syndrome, obesity is often associated with this peripheral neuropathy. Fatty deposition in patients with obesity and metabolic syndrome is the major cause of nerve injury as it may deposit in extracellular protein glycation, nerves, oxidative stress, and mitochondrial dysfunction. ${ }^{26}$

Obese individuals may also develop other comorbidities, this may lead to the formation of edema in the extremity and later on may worsen the median nerve compression. $^{27}$ The peak incidence of CTS is around 55-66 years old individuals. Obesity could also develop edema that sufficient to compress the median nerve and increase the carpal tunnel pressure even in adolescent individuals. ${ }^{28-30}$ According to a meta-analysis which was conducted by Shiri et al., men with obesity tend to generate a 1.5 times bigger risk of acquiring CTS than slender individuals. The study also states that every unit's increasement in BMI intensifies the risk of CTS by $7.4 \%$ as it is causing an unfavorable defense against biomechanical exposures. ${ }^{26,31}$ Short and wide hands are more vulnerable to acquire CTS. Square-shape of the hand may also generate more risk in developing adverse effects caused by obesity. ${ }^{31}$

\section{Association between Age and CTS}

Although the natural history of CTS with associated risk factors is poorly understood. This focal compressive neuropathy and demyelination could deteriorate over time. According to this concept, it is possible to find a positive correlation between complaint and severity with increasing age. Increased in distal or sensory latencies is known to be caused aging factor. It is stated that age and anthropometric measurements contribute to increasing the median and ulnar nerve latencies. However, this might also be affected by factors other than the aging process. Age-specific incidence in a woman is increasing analogous with age especially at the sixth decade and declining afterward. ${ }^{30,32}$

The incidence rate of CTS itself showed that the elderly tend to form more CTS compared to younger patients. ${ }^{30}$ Severity of the CTS is also higher in elderly patients based on clinical signs, electrophysiology, and ultrasound examination. Many studies have shown of age contributes significantly to forming CTS. $^{32}$

\section{Association between gender and CTS}

There are different results in CTS incidence among men and women. Women are likely to develop CTS three times higher than men. Several factors are contributing to this condition, starting from hormonal status, pregnancy, anthropometric properties, biomechanical exposures. ${ }^{33} \mathrm{~A}$ study conducted among industrial workers with the exposure of biomechanical work, women had a 2 times higher chance to acquire CTS. ${ }^{34}$ Endocrine factors could play a major role in generating CTS. Besides hormonal factors in pregnancy, menopause has also been thought to be a risk factor. ${ }^{33}$ Idiopathic CTS in women may also be caused by estrogen receptors upregulation within the tenosynovial tissue. This may contribute to fluid retention and hormonal fluctuation which are resulting in local edema and lead to alteration of spaces ratio within the carpal tunnel. ${ }^{35}$ According to a case-control in Turkey, the age of menopause can be used as a predictor of CTS formation. ${ }^{36}$

Distinctive

anthropometric properties may also be in charge in association with CTS. The different configurations of each gender's wrist and carpal tunnel anatomy predispose the CTS condition. Smaller free space in the tunnel was also proven by magnetic resonance imaging in a study focused on the characteristic of women's carpal tunnel. The condition could lead to a higher risk of CTS 
and also a more severe CTS condition. ${ }^{37}$ In addition, full-time housewives were found to be having a higher incidence of CTS compared to non-manual workers, repetitive activities of biomechanical exposure were responsible for this. ${ }^{38}$

\section{Occupational risk factor}

The constraint spaces inside the tunnel with median nerve and nine flexor tendons pass through the tunnel cause the carpal tunnel to be vulnerable against several hand motions. ${ }^{14}$ Physical occupation activities, such as repeated and forceful posture changes of the wrist are quite commonly associated with CTS. Routine and prolonged flexion or extension of the wrist are highly related to the drastic changes in the tunnel's pressure. ${ }^{29}$ Fish and meat processing industry workers with workers in the forestry industry were found to have the highest risk of developing CTS. It is thought that a minimum of $4 \mathrm{~kg}$ hand force with more than $50 \%$ repeated performing the same motion or cycle time under 10 seconds, and an 8 hours frequent movement with an acceleration of $3,9 \mathrm{~m} / \mathrm{s}^{2}$. Exposure to prolonged strong vibration may also increase the risk of CTS, for example, workers that need to use rock drill machines and chainsaws. ${ }^{29,39}$

\section{Multifactorial Contributions in CTS}

CTS is a multifactorial condition that occurs with a combination of several risk factors. In the past, CTS was thought to be caused by a single risk factor related to repetitive hand and wrist movements, especially individuals that spend most of their work with their computer. ${ }^{15}$ However, many predisposition conditions turned out to affect the occurrence of CTS. For example, a congenital predisposition is highly related to CTS cases. Any comorbidities may also contribute to the making of CTS it shows that CTS is a multifactorial condition that could not be explained as caused by only a single risk factor. There is barely any study research that stands with CTS as a single risk factor condition. ${ }^{10,15,24}$ This multifactorial aspect can be used to implicate the treatment for those with CTS. Eliminating the risk factor hopefully may decrease the severity of the condition parallel with musculoskeletalspecific treatments. Before taking the patients into surgery, non-surgical therapy should be applied at first, such as medications, wrist splints, physical therapy, and reducing the risk factors. Alternative working tools at work can be protective equipment regarding the biomechanical exposure from vibration and forceful movements of the wrist. ${ }^{15,40}$ Not only can risk factors be implied in the therapeutic approach, but also in becoming a CTS predictor. Any protective equipment including avoidance of the risk factor can be considered.

\section{CONCLUSION}

CTS is one of the most common peripheral neuropathies. Until now, the exact progress is still unknown; several risk factors may contribute to forming CTS. Increased pressure inside the carpal tunnel could compress the median nerve causing several disturbances starting from mild to severe symptoms. CTS is a multifactorial condition that is caused by the association of several risk factors that may also contribute to the severity of the condition. It is important for clinicians to know and notice the risk factors as it may reduce the chance of acquiring CTS. By knowing the risk factors of CTS, clinicians will have the option to treat the patients regarding the implications of the risk factor in reducing the symptoms and severity of CTS. Further studies need to be conducted to study the effect of a single risk factor contribution in the making of CTS.

\section{Acknowledgement: None}

Conflict of Interest: None

Source of Funding: None

Ethical Approval: Not Applicable 


\section{REFERENCES}

1. Atroshi I, Gummesson C, Johnsson R, Ornstein E, Ranstam J, Rosen I. Prevalence of carpal tunnel syndrome in a general population. Jama. 1999 Jul 14;282(2):153-8.

2. Burton CL, Chen Y, Chesterton LS, van der Windt DA. Trends in the prevalence, incidence and surgical management of carpal tunnel syndrome between 1993 and 2013: an observational analysis of UK primary care records. BMJ open. 2018 Jun 1;8(6):e020166.

3. Papež BJ, Palfy M, Turk Z. Infrared thermography based on artificial intelligence for carpal tunnel syndrome diagnosis. Journal of International Medical Research. 2008 Dec;36(6):1363-70.

4. Amirlak B, Upadhyaya K, Ahmed O, Wolff T, Tsai T, Scheker L. Median Nerve Entrapment. Internet Communication. 2010:1-1

5. Burton C, Chesterton LS, Davenport G. Diagnosing and managing carpal tunnel syndrome in primary care. British Journal of General Practice. 2014 May 1;64(622):2623.

6. Chammas M, Boretto J, Burmann LM, Ramos RM, Santos Neto FC, Silva JB. Carpal tunnel syndrome-Part I (anatomy, physiology, etiology and diagnosis). Revista brasileira de ortopedia. 2014 Oct;49(5):42936.

7. Kothari MJ. Carpal tunnel syndrome: Etiology and epidemiology. Uptodate. com. 2014.

8. Roel-Valdés J, Arizo-Luque V, RondaPérez E. Epidemiology of occupationallycaused carpal tunnel syndrome in the province of Alicante, Spain 1996-2004. Revista espanola de salud publica. 2006;80(4):395-409.

9. Jenkins PJ, Watts AC, Duckworth AD, McEachan JE. Socioeconomic deprivation and the epidemiology of carpal tunnel syndrome. Journal of Hand Surgery (European Volume). 2012 Feb;37(2):123-9.

10. Raja S, Carlos AJ. Carpal tunnel syndromecurrent controversies. Orthopaedics and Trauma. 2015 Aug 1;29(4):273-7.

11. Komurcu HF, Kilic S, Anlar O. Relationship of age, body mass index, wrist and waist circumferences to carpal tunnel syndrome severity. Neurologia medicochirurgica. 2014;54(5):395-400.
12. Ebrahimzadeh MH, Mashhadinejad H, Moradi A, Kachooei AR. Carpal tunnel release in diabetic and non-diabetic patients. Archives of Bone and Joint Surgery. 2013 Sep;1(1):23.

13. Chammas M, Boretto J, Burmann LM, Ramos RM, Santos Neto FC, Silva JB. Carpal tunnel syndrome-Part I (anatomy, physiology, etiology and diagnosis). Revista brasileira de ortopedia. 2014 Oct;49(5):42936.

14. Ghasemi-Rad M, Nosair E, Vegh A, Mohammadi A, Akkad A, Lesha E, Mohammadi MH, Sayed D, Davarian A, Maleki-Miyandoab T, Hasan A. A handy review of carpal tunnel syndrome: From anatomy to diagnosis and treatment. World journal of radiology. 2014 Jun 28;6(6):284.

15. Ibrahim I, Khan WS, Goddard N, Smitham P. Suppl 1: carpal tunnel syndrome: a review of the recent literature. The open orthopaedics journal. 2012;6:69.

16. Millesi H, Zöch G, Rath TH. The gliding apparatus of peripheral nerve and its clinical significance. InAnnales de Chirurgie de la Main et du Membre Superieur 1990 Jan 1 (Vol. 9, No. 2, pp. 87-97). Elsevier Masson.

17. Duncan SF, Bhate O, Mustaly $\mathrm{H}$. Pathophysiology of carpal tunnel syndrome. InCarpal Tunnel Syndrome and Related Median Neuropathies 2017 (pp. 13-29). Springer, Cham.

18. Aboonq MS. Pathophysiology of carpal tunnel syndrome. Neurosciences (Riyadh). 2015;20(1):4-9.

19. Werner RA, Andary M. Carpal tunnel syndrome: pathophysiology and clinical neurophysiology. Clinical Neurophysiology. 2002 Sep 1; 113(9): 1373-81.

20. Alfonso C, Jann S, Massa R, Torreggiani A. Diagnosis, treatment and follow-up of the carpal tunnel syndrome:

21. Frieboes LR, Palispis WA, Gupta R. Nerve compression activates selective nociceptive pathways and upregulates peripheral sodium channel expression in Schwann cells. Journal of Orthopaedic Research. 2010 Jun;28(6):753-61.

22. Aroori S, Spence RA. Carpal tunnel syndrome. Ulster Med J. 2008;77(1):6-17.

23. Rempel D, Evanoff B, Amadio PC, De Krom M, Franklin G, Franzblau A, Gray R, Gerr F, Hagberg M, Hales T, Katz JN. Consensus criteria for the classification of carpal tunnel syndrome in epidemiologic 
studies. American journal of public health. 1998 Oct;88(10):1447-51.

24. MacDermid JC, Doherty T. Clinical and electrodiagnostic testing of carpal tunnel syndrome: a narrative review. Journal of Orthopaedic \& Sports Physical Therapy. 2004 Oct;34(10):565-88.

25. Shiri R, Pourmemari MH, Falah-Hassani K, Viikari-Juntura E. The effect of excess body mass on the risk of carpal tunnel syndrome: a meta-analysis of 58 studies. Obesity Reviews. 2015 Dec;16(12):1094-104.

26. Callaghan B, Feldman E. The metabolic syndrome and neuropathy: therapeutic challenges and opportunities. Annals of neurology. 2013 Sep;74(3):397-403.

27. Werner R, Armstrong TJ, Bir C, Aylard MK. Intracarpal canal pressures: the role of finger, hand, wrist and forearm position. Clinical Biomechanics. 1997 Jan 1;12(1): 44-51.

28. Tseng CH, Liao CC, Kuo CM, Sung FC, Hsieh DP, Tsai CH. Medical and nonmedical correlates of carpal tunnel syndrome in a Taiwan cohort of one million. European journal of neurology. $2012 \mathrm{Jan} ; 19$ (1):91-7.

29. Burt S, Crombie K, Jin Y, Wurzelbacher S, Ramsey J, Deddens J. Workplace and individual risk factors for carpal tunnel syndrome. Occupational and environmental medicine. 2011 Dec 1;68 (12):928-33.

30. Mondelli M, Giannini F, Giacchi M. Carpal tunnel syndrome incidence in a general population. Neurology. 2002 Jan 22;58 (2):289-94.

31. Shiri R. A square-shaped wrist as a predictor of carpal tunnel syndrome: A meta-analysis. Muscle \& nerve. 2015 Nov;52(5):709-13.1

32. Kouyoumdjian JA. Carpal tunnel syndrome: age, nerve conduction severity and duration of symptomatology. Arquivos de neuropsiquiatria. 1999 Jun;57(2B):382-6.

33. Padua L, Pasquale AD, Pazzaglia C, Liotta GA, Librante A, Mondelli M. Systematic review of pregnancy-related carpal tunnel syndrome. Muscle \& nerve. 2010 Nov;42 (5):697-702.
34. Bianchi SM, Milkie MA, Sayer LC, Robinson JP. Is anyone doing the housework? Trends in the gender division of household labor. Social forces. 2000 Sep 1;79(1):191-228.

35. Kim JK, Hann HJ, Kim MJ, Kim JS. The expression of estrogen receptors in the tenosynovium of postmenopausal women with idiopathic carpal tunnel syndrome. Journal of Orthopaedic Research. 2010 Nov;28(11):1469-74.

36. Kaplan Y, Kurt SG, Karaer H. Carpal tunnel syndrome in postmenopausal women. Journal of the neurological sciences. 2008 Jul 15;270(1-2):77-81.

37. Bower J, Stanisz G, Keir P. An MRI evaluation of carpal tunnel dimensions in healthy wrists: Implications for carpal tunnel syndrome. Clinical Biomechanics. 2006;21(8):816-825.

38. Mattioli S, Baldasseroni A, Curti S, Cooke RM, Mandes A, Zanardi F, Farioli A, Buiatti E, Campo G, Violante FS. Incidence rates of surgically treated idiopathic carpal tunnel syndrome in blue-and white-collar workers and housewives in Tuscany, Italy. Occupational and Environmental Medicine. 2009 May 1;66(5):299-304.

39. Tanaka S, Wild DK, Cameron LL, Freund E. Association of occupational and nonoccupational risk factors with the prevalence of self-reported carpal tunnel syndrome in a national survey of the working population. American Journal of Industrial Medicine. 1997 Nov;32(5):550-6.

40. Mansoor S, Siddiqui M, Mateen F, Saadat S, Khan ZH, Zahid M, Khan HH, Malik SA, Assad S. Prevalence of Obesity in Carpal Tunnel Syndrome Patients: A CrossSectional Survey. Cureus. 2017 Jul;9(7).

How to cite this article: Meregawa PF, Nolan J. The role of multifactorial contribution in carpal tunnel syndrome occurrence. International Journal of Science \& Healthcare Research. 2021; 6(2): 266-272. DOI: https://doi.org/ 10.52403/ijshr.20210447 